\title{
Yield Modeling of Acoustic Charge Transport Transversal Filters
}

\author{
J. Stevenson Kenney, Member, IEEE, Gary S. May, Member, IEEE, and William D. Hunt, Member, IEEE
}

\begin{abstract}
This paper presents a yield model for acoustic charge transport transversal filters. This model differs from prevlous IC yield models in that it does not assume that individual failures of the nondestructive sensing taps necessarily cause a device failure. A redundancy in the number of taps included in the design is explained. Poisson statistics are used to describe the tap failures, weighted over a uniform defect density distribution. A representative design example is presented. The minimum number of taps needed to realize the filter is calculated, and tap weights for various numbers of redundant taps are calculated. The critical area for device failure is calculated for each level of redundancy. Yield is predicted for a range of defect densities and redundancies. To verify the model, a Monte Cario simulation is performed on an equivalent circuit model of the device. The results of the yield model are then compared to the Monte Carlo simulation. Better than $95 \%$ agreement was obtained for the Poisson model with redundant taps ranging from $30 \%$ to $150 \%$ over the minimum.
\end{abstract}

\section{INTRODUCTION}

A COUSTIC charge transport (ACT) devices are charge transfer devices similar to conventional charge coupled devices (CCD), except that the mechanism for localizing and transporting charge packets is an electric field induced in a piezoelectric material by a surface acoustic wave (SAW) [1]. The basic operation of the ACT device consists of input sampling, charge transport, and output sensing. Since nondestructive sensing is used, multiple outputs can be summed at various locations along the transport channel. In this configuration, the device operates as a tapped delay line. Since it is fabricated using GaAs heterostructures, MESFET's and other devices can be easily fabricated on integrated circuits to provide tap weighting and amplification. If the weighted taps are summed on a common output bus, a programmable transversal filter (PTF) structure is obtained [1]. A PTF block diagram is shown in Fig. 1.

Transversal filters are used frequently in digital signal processing (DSP) at much lower frequencies and bandwidths. ACT-PTF's are roughly three orders of magnitude faster than conventional DSP technology due to the fact that multiply and accumulate functions are done in parallel by analog circuits. SAW devices can also perform the PTF function. Their primary disadvantage is that they are not made with semiconducting materials, and thus external circuitry cannot be integrated in monolithic form. Si CCD technology was also once used for PTF's at lower frequencies [2]. However,

Manuscript received November 8, 1993; revised July 4, 1994. This work was supported by NASA under Contract NAGW-2753.

The authors are with the School of Electrical and Computer Engineering. Georgia Institute of Technology. Allanta, GA 30332-0250 USA

IEEE Log Number 9409819

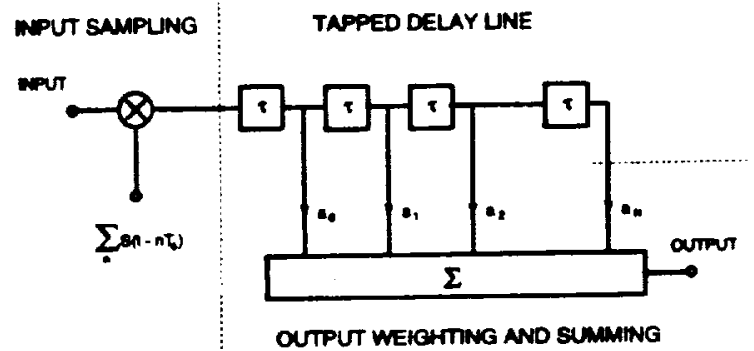

Fig. 1. Block diagram of an ACT-PTF.

because of inherent speed limitations in MOSFET charge transfer devices, DSP technology has made this approach obsolete. High speed GaAs CCD's show potential for this application, but devices with sufficient delay have not yet been demonstrated.

ACT device technology was first demonstrated by Gaalema et al. in 1976 [3]. This device relied on the potential induced in a piezoelectric material in close contact with a depleted silicon metal-insulator-semiconductor (MIS) capacitor to transport injected charge at the acoustic velocity. In 1982, Hoskins et al. reported the first monolithic ACT using GaAs technology [4]. GaAs is both semiconducting and weakly piezoelectric, allowing both the acoustic transducer and the depletion region to be fabricated on the same die. In 1987, Tanski reported the first GaAs/AlGaAs heterostructure ACT (HACT) device [5]. Today, ACT devices are in low-volume production for radar signal processing and other high-speed signal processing applications [6]. Other high volume commercial applications, such as computer disk drive equalization and imaging, are also being investigated. Such high-volume applications will require significant improvements in the production yield.

ACT device yield depends on many parameters. Material defects affect the yield in a global sense with both catastrophic and parametric failures. In addition to material defects, spot defects due to lithography faults cause additional catastrophic failures. Since material defects cannot be designed out, improvements in yield will be obtained only from careful control of the manufacturing process. Like material defects, yield can be improved through process control. However, because of the large die size $\left(\sim 1 \mathrm{~cm}^{2}\right.$, typically), additional improvements may be required for commercial feasibility. In a previous paper, we presented an approach to improve yield, which is implemented in the design phase through the use of redundant circuits [7]. This paper expands upon this in several ways. The minimum number of taps to theoretically meet a filter specification is calculated. From this, a number of designs 


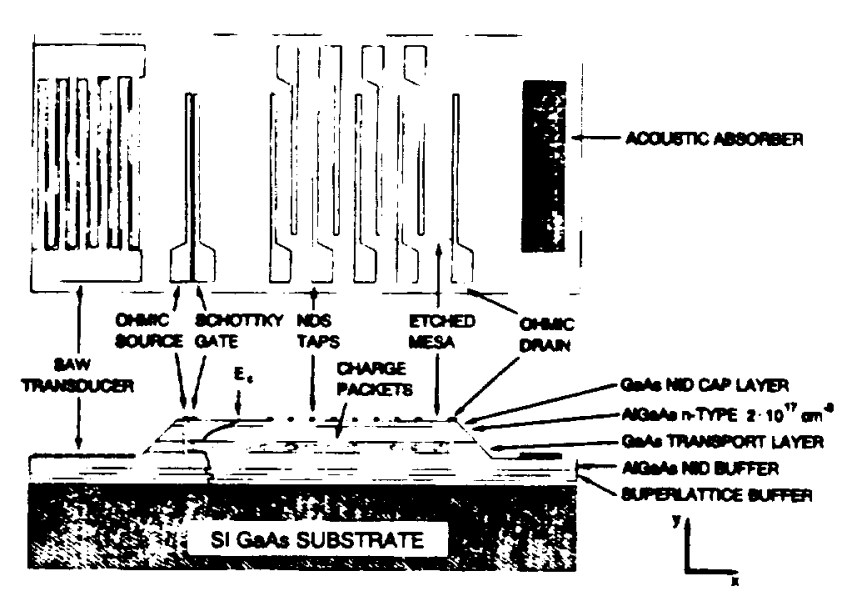

Fig. 2. Schematic representation of the physical structure of an ACT-PTF.

are developed around a given redundancy factor. This factor is then included in the yield model, which is based on a cumulative Poisson function. In [7], we showed that uniformly distributed defect densities provide the most accurate yield predictions. Yield predictions are performed for PTF's with $30 \%-150 \%$ redundancy over the minimum number of taps (i.e., a range of redundancy factors from 1.3 to 2.5 ). The model is then verified by performing a Monte Carlo yield simulation on equivalent circuit models of each of the filters. The basis of the equivalent circuit models is given in [8].

\section{ACT PTF CIRCUIT MODEL}

The physical structure of a HACT device is shown schematically in Fig. 2. The charge injection is accomplished by a MESFET. Mobile electrons in the source region are swept through the depletion region by the SAW potential created by the acoustic transducer. As the SAW potential sweeps beneath a gate electrode, a discrete packet of charge breaks off and is transported down the ACT channel at the acoustic velocity. Since the depth of the depletion region is modulated by the gate potential, the amount of charge in the packet is proportional to the input signal. The equivalent circuit that represents this operation is a voltage-controlled current source, with the value of the transconductance being averaged over one SAW period. This is shown in Fig. 3, along with the remainder of the ACT-PTF equivalent circuit model developed in [8]. The SAW propagates as a guided wave, and is modeled as a transmission line with a phase velocity equal to the acoustic velocity. The ACT channel is periodically tapped by sensing elements at the surface of the channel. These elements are reversed-biased Schottky diodes. The charge packets moving beneath the taps create an image current in the Schottky metal proportional to the channel current. This is modeled by the Noron equivalent circuit. Tap weighting is accomplished by a capacitive current divider, and the sign can be implemented using a differential amplifier (not shown).

As shown in [8], the effects of the parasitic elements, $R_{G}, C_{G S}$, and $R_{O}$, the nonzero width sampling aperture, and the frequency response of the differential amplifier can be neglected. The frequency response of the ACT-PTF is determined by the ratios of the weighting capacitors $C_{i}$, to
TRWNSFER CIRCUIT

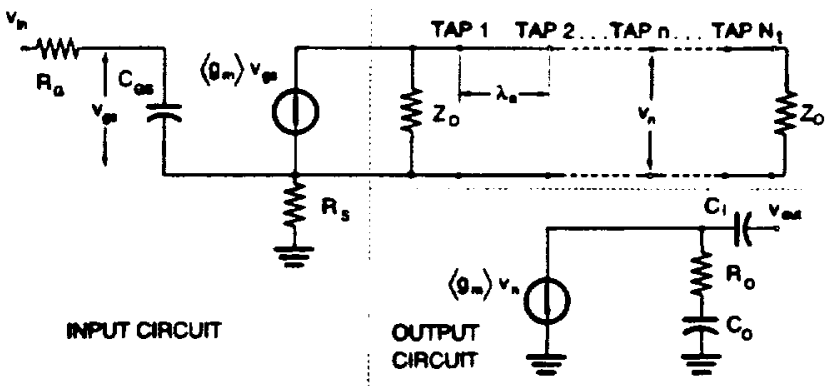

Fig. 3. An equivalent circuit for an ACT-PTF showing a single output circuit. Tap weighting is accomplished by the capacitors $C$, of multiple output circuits.

TABLE I

Design SPECITICATION por a BandPASS FLter

\begin{tabular}{|c|c|c|}
\hline Cenver frequency $(V J$ : & & $70.0 \mathrm{MHz}$ \\
\hline Equiripple Bendwidth [D]: & & $20 \mathrm{MHz}$ \\
\hline Puscand Ripple: & & $\pm 0.5 \mathrm{~dB}$ \\
\hline Ou-of-Band Rejaction: & $\begin{array}{l}10-55 \mathrm{MHz} \\
\mathrm{s}-130 \mathrm{MHz}\end{array}$ & $\begin{array}{l}2506 \\
2568\end{array}$ \\
\hline
\end{tabular}

the output capacitance $C_{O}$. The ACT-PTF output current io is given by

$$
\begin{aligned}
i_{O} & =\left\langle g_{m}\right\rangle \sum_{i=1}^{N_{i}} \nu_{g s}\left(t-i T_{s}\right) \cdot s_{i} \frac{C_{i}}{C_{O}+C_{i}} \\
& \approx\left\langle g_{m}\right\rangle \sum_{i=1}^{N_{i}} \nu_{g s}\left(t-i T_{s}\right) \cdot s_{i} \frac{C_{i}}{C_{O}}, \quad \text { for } C_{i} \ll C_{O}
\end{aligned}
$$

where $\left\langle g_{m}\right\rangle$ is the sample-averaged transconductance, $N_{t}$ is the number of taps, $\nu_{g a}$ is the input signal, $T_{s}$ is the sampling period, and $s_{i}$ is the tap sign created by the differential amplifier. This is essentially the form of the impulse response of the transversal filter shown in Fig. 1. What distinguishes this type of filter architecture from traditional analog filters is the fact that the impulse response is finite. Since this type of filter is commonly used in DSP, methods have been developed to synthesize the tap weights of such a filter [9]. We will choose a representative design example in order to investigate the yield properties of ACT-PTF's. A typical design specification for this example is shown below in Table $I$.

One design approach for FIR filiers is to assume an ideal square passband, and calculate the tap coefficients from wellknown Fourier transform relationships [10]. The ith tap weight is given as

$$
w_{i}=\frac{\sin \left[\pi B T_{a}\left(i-N_{t} / 2\right)\right]}{\pi B T_{0}\left(i-N_{t} / 2\right)} \cdot \cos (i \pi)
$$

where $T_{0}$ is the Nyquist sampling period $\left(=1 / 2 f_{c}\right)$, and $N_{t}$ is the number of taps, and $B$ is the bandwidth parameter. The rectangular window function inherent in (2) results in degraded stopband attenuation. Because the tap truncation is essentially a frequency domain convolution with a $\sin (f) / f$ function, thr stopband attenuation is limited to about $20 \mathrm{~dB}$. Various methods have been proposed to circumvent this limitation [9], 
and all of these rely on multiplying the tap weight sequence by a function that descends to zero in a more gradual manner than the rectangular window. One of the more common window functions is the Kaiser window [9]:

$$
K(n)=\frac{I_{0}\left(\beta \sqrt{\left[1-\left(\frac{n-N_{t} / 2}{N_{t} / 2}\right)\right]^{2}}\right)}{I_{0}(\beta)}
$$

for $0 \leq i \leq N_{t}$, where $I_{0}$ is the modified Bessel function of the first kind, and $\beta$ is a shape parameter determined by the stopband ripple $\delta$,

$$
\beta=\left\{\begin{array}{cl}
0.1102(A-8.7) & A>50 \\
0.5842(A-21)^{0.4} & \\
\quad+0.07886(A-21) & 21 \leq A \leq 50 \\
0 & A<21
\end{array}\right.
$$

where

$$
A=20 \log \delta .
$$

Thus we arrive at the formula for the tap weighting capacitors $C_{i}$ :

$$
C_{i}=w(n) K(n) C_{\mathrm{t}}
$$

where $C_{t}$ is a fixed value such that $C_{t} \ll C_{O}$. The sign of the weighing function is implemented with a switch and differential amplifier circuit not explicitly included in this circuit model. For the purposes of yield prediction, we simply include the sign of $C_{i}$ in the element value.

Kaiser has also provided a semj-empirical expression to predict the minimum number of taps for a given filter response based on passband flatness $\delta_{1}$ stopband ripple $\delta_{2}$, and transition bandwidth $\Delta f[9]$.

$$
N_{\min }=\frac{-10 \log \left(\delta_{1} \delta_{2}\right)-13}{14.6 \Delta f T_{s}}
$$

However, parasitic effects tend to dominate the fine-grain behavior to ACT-PTF's and reduce yield. For this reason, it is common to include a redundancy factor $r$, such that $N_{t}=$ $r N_{\min }$, to provide for performance margin over the specified flatness, rejection, and transition bandwidth. Too many redundant taps, however, results in a larger die size, and hence a lower yield. We investigate herein a range of redundancy factors from $r=1.3$, to $r=2.5$. The lower bound of this range was empirically determined to be the minimum for the given specification. The upper bound was also determined empirically to be the highest feasible factor that could result in yield improvement. This range results in the number of taps being extended from 29 to 71 . The simulated nominal frequency response of this ACT-PTF is shown in Fig. 4.

\section{ACT-PTF YIELD MODEL DEVELOPMENT}

This paper concentrates on ACT-PTF failures due to missing taps. This is a common failure dependent on a number of defects [5]. Photoresist defects can result in metal being missing from some portion of the tap. In addition to this,

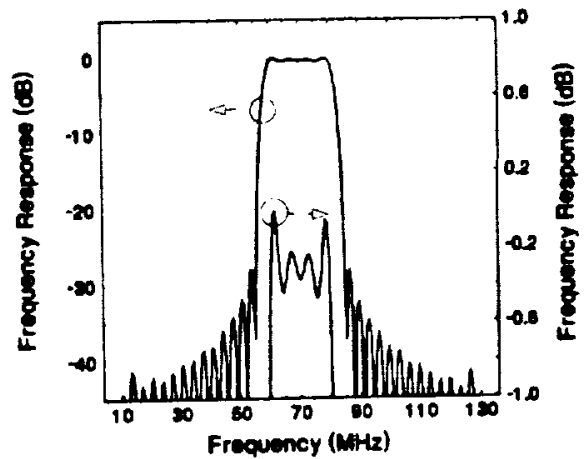

Fig. 4. Nominal frequency response of an ACT.PTF designed to meet the specification given in Table I. The redundancy of this filter is $r=1.5$.

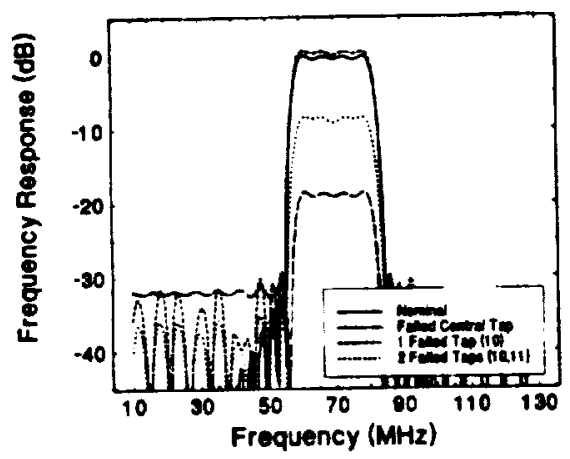

Fig. 5. Effect of tap failures on the frequency response of an ACT-PTF.

tap weighting and interconnect circuits can also contribute to tap failures. However, unlike digital circuits and most analog circuits, a missing tap circuit does not necessarily result in a device failure. This soft failure mode is due to the parallel operation of the device. Thus, when the circuit is designed with some redundancy factor greater than unity, effective redundancy in the yield performance results. Fig. 5 shows the effect of missing taps on the frequency response. This figure shows the nominal response, and three responses degraded due to missing tap circuits. The one showing the worst rejection is the result of removing the middle tap (at the peak of the $\sin (x) / x$ ). Taps are "removed" by setting the appropriate weighting capacitor $C_{i}$ to zero. The next worst rejection is caused by removing two taps about mid-way off of the center of the impulse response function. The last degraded trace actually meets the design specification, and results from removing a single tap away from the center for the impulse response. From this data, one might infer that an effective redundancy of one tap exists. Our goal is to determine to what extent this is true, and to make yield predictions for a given process.

Previous yield models that have been developed have concentrated mostly on the yield of digital VLSI circuits, or other such circuits whereby single circuit failure results in a bad die [12], [15]. As shown above, this is somewhat pessimistic for the ACT-PTF. It is possible, however, to extend some of these models to account for the effective redundancy. The Poisson model was chosen as a basis for the redundant-tap yield model for the following reasons:

1) It has been shown to be accurate for spot defects with relatively low defect densities [13]. 


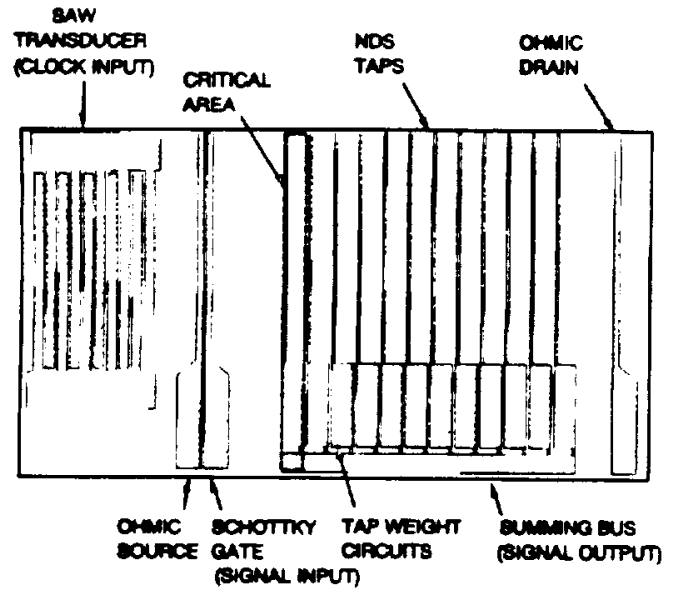

Fig. 6. Critical area of the tap circuits used in the yield prediction.

2) It is derived from first principles of statistical failure analysis (i.e., the limit of the binomial distribution for small average failure rates) [14].

3) It does not require a priori knowledge of empirically determined process parameters, other than defect density.

4) It is simple in form and easy to modify to account for redundancy.

The basic form of a Poisson model modified for redundancy is simply the cumulative Poisson function calculated over some defect density distribution function:

$$
Y_{m}=\sum_{i=0}^{m} \int_{0}^{\infty} \frac{\left(N_{t} A_{c} D\right)^{i} \exp \left(-N_{t} A_{c} D\right)}{t !} f(D) d D
$$

where $A_{c}$ is the critical area of the tap and weighting circuitry, $D$ is the defect density, and $f(D)$ is the defect density distribution function. The critical area is calculated for a heterojunction ACT device currently being fabricated at Georgia Tech is shown in Fig. 6. The width of the tap fingers is usually quite small $(2 \mu \mathrm{m})$. However, since the defect size is not specified for this model, it is assumed that a defect occurring anywhere within one half acoustic wavelength $(20 \mu \mathrm{m}$ at the operating frequency) causes a tap failure. This is a conservative estimate, but this model could be refined if the distribution of defect sizes was known [12]. The channel width, and hence the tap length is $1 \mathrm{~mm}$. The interconnect and weighting circuits also take up approximately $50 \%$ more area. This yields an effective critical area per tap circuit of

$$
\begin{aligned}
A_{c} & =(20 \mu \mathrm{m})(1000 \mu \mathrm{m})(1.5) \\
& =3.0 \cdot 10^{-4} \mathrm{~cm}^{2} .
\end{aligned}
$$

In [7], we investigated three defect density distribution functions: delta function, uniform, and triangular (see Fig. 7). These are given below in $(9(\mathrm{a}))-(9(\mathrm{c}))$, respectively:

$$
\begin{aligned}
& f(D)=\delta\left(D_{0}\right) \\
& f(D)= \begin{cases}\frac{1}{2 D_{0}} & 0 \leq D \leq 2 D_{0} \\
0 & D>2 D_{0}\end{cases}
\end{aligned}
$$

(D)

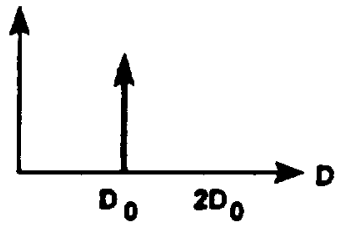

(a)

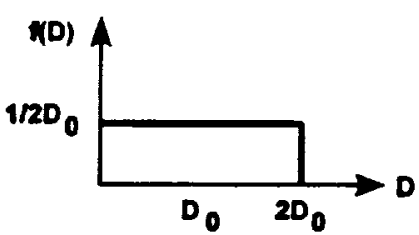

(b)

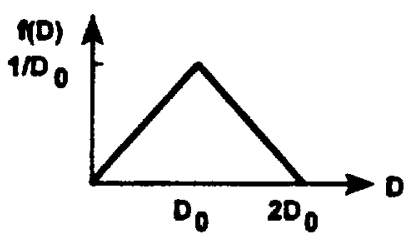

(c)

Fig. 7. Defect probability density functions [15]. (2) Delu function distribution. (b) Uniform distribution. (c) Triangle function distribution.

$$
f(D)= \begin{cases}\frac{D}{D_{0}^{2}}, & 0 \leq D \leq D_{0} \\ \frac{2 D_{0}-D}{D_{0}^{2}}, & D_{0}<D \leq 2 D_{0} \\ 0, & D>2 D_{0}\end{cases}
$$

where $D_{0}$ is the average defect density of the given distribution. It was found that the uniform defect density distribution (9(b)) provided the best fit to the yield as predicted by the Monte Carlo model. Substituting this into (6), we obtain an analytic yield model $Y_{u, m}\left(D_{0}\right)$

$Y_{u, m}\left(D_{0}\right)=\sum_{i=0}^{m} \frac{1}{2 D_{0}} \int_{0}^{2 D_{0}} \frac{\left(N_{t} A_{c} D\right)^{i} \exp \left(-N_{t} A_{c} D\right)}{i !} d D$

It was also shown in [7] that the one redundancy model $(m=1)$ is in the best agreement with the expected yield. We calculated the yield model for a range of average defect densities $D_{0}$ for the $m=1$ case. The results of these calculations are shown in Fig. 8(a)-(d), along with the results from the Monte Carlo simulations discussed in the next section.

\section{Monte Carlo Yield Simulation}

To establish the validity of the one-redundancy Poisson models developed in the last section, a Monte Carlo yield analysis was performed on the ACT-PTF equivalent circuit model. Essentially, this involves calculating the frequency response a number of times with the tap capacitors weighted by a binomial random variable $b \in\{0,1\}$, with a probability of failure $p$. Monte Carlo yield estimation is included in Libra. ${ }^{\mathrm{TM}}$

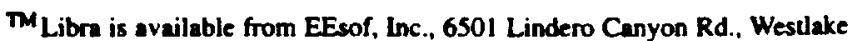
Village, CA 91362
} 


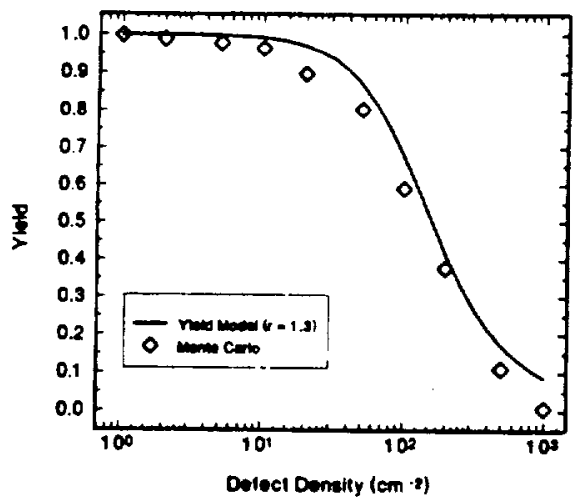

(a)

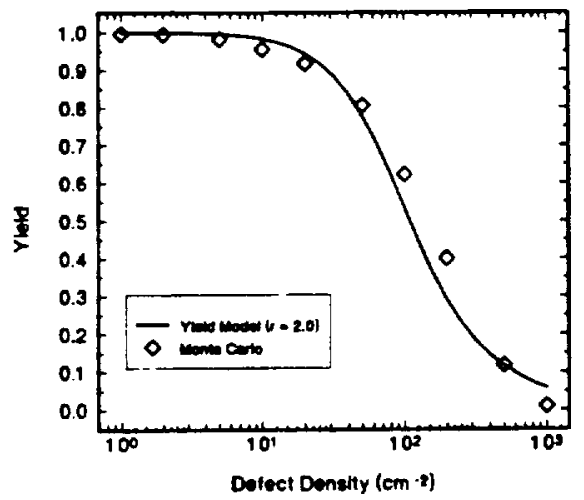

(c)

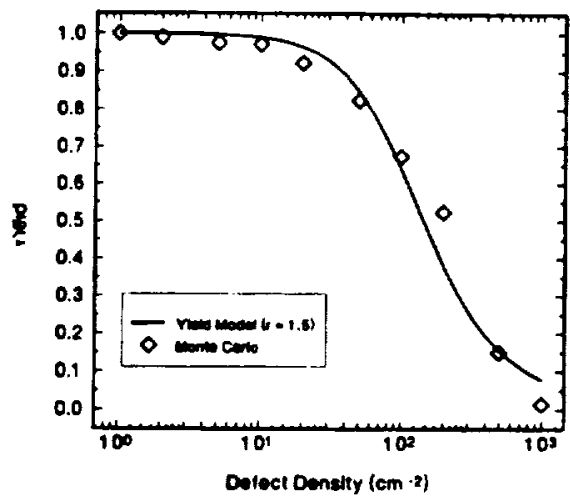

(b)

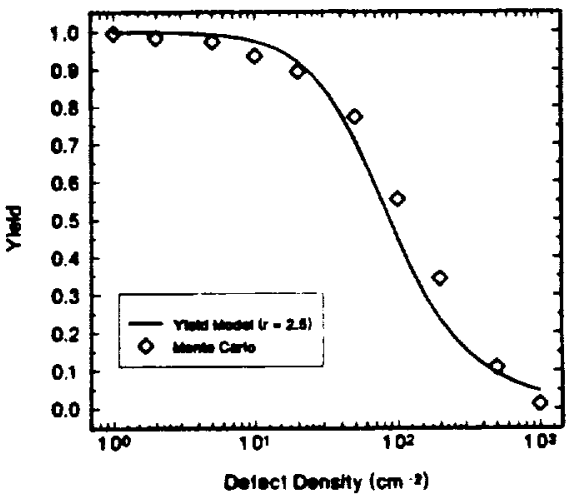

(d)

Fig. 8. Yield model calculations given by (10) (solid line) compared to Monte Carlo predictions $(\diamond)$. (a) $r=1.3$. (b) $r=$ 1.5. (c) $r=2.0$. (d) $r=2.5$.

However, because it is an analog simulator, binomial random variables are not supported. In order to calculate the yield due to catastrophic failures of individual taps, a way to simulate the binomial random variables was devised by creating a binomial random variable $b$ from the transformation of a continuous random variable $x \in[0,1]$. One such transformation is:

$$
b=\frac{1}{2}[\tanh (\alpha(x-1+\mu))]
$$

where $\alpha$ is an empirical weighting parameter, $\mu$ is the expected value of $b$, and is related to the probability of tap failure $p$ by $\mu$ $=1-p$. When $\alpha$ is large (we used $\alpha=100$ ), the mapping causes the value of $b$ to shift abruptly from 0 to $l$ when $\alpha$ is in the vicinity of $\mu$. Hence $b$ is approximately binomial $(b \in\{0,1\})$.

The only parameter required for Monte Carlo yield simulation is the number of trials $N$. This was chosen by requiring a $99 \%$ probability of at least one tap failing for the lowest defect density. This is evaluated from the Poisson distribution in the following manner:

$$
\begin{gathered}
P(y \geq 1)=1-P(y=0)=1-\exp \left(N A_{\mathrm{c}} D\right)=0.99 \\
\Rightarrow N=384 \quad \text { for } D=1 \mathrm{~cm}^{-2} .
\end{gathered}
$$

To be conservative, this number was rounded up to $N=500$. The simulation results are shown in Fig. 8(a)-(d). The Monte Carlo simulation for each defect density look approximately 15 min on a Sun Sparc 10 running Libra ${ }^{\mathrm{TM}}$, version 3.5, sweeping 107 frequency points. The Libra ${ }^{\mathrm{TM}}$ Monte Carlo simulation is

\begin{tabular}{|c|c|c|c|c|}
\hline \multirow{2}{*}{$\begin{array}{l}\text { Defed } \\
\text { Deredy }\left(\cos ^{-1}\right)\end{array}$} & \multicolumn{4}{|c|}{ Preclleded Yinded } \\
\hline & $r=1.3$ & $r=1.5$ & $r=2.0$ & $r=2.5$ \\
\hline 1 & .998 & 1.000 & .996 & $.0 \%$ \\
\hline 2 & .988 & .90 & .994 & .984 \\
\hline$s$ & .978 & .976 & .982 & .974 \\
\hline 10 & .964 & 972 & .956 & .936 \\
\hline 20 & $.8 \%$ & .922 & .918 & .894 \\
\hline 50 & .002 & .204 & $\infty$ & $\pi$ \\
\hline 100 & .590 & .674 & .622 & .344 \\
\hline 200 & .378 & .524 &.$\infty 0$ & .342 \\
\hline 300 & .110 & .150 & .118 & .106 \\
\hline 1000 & .006 & .014 & .010 & .012 \\
\hline
\end{tabular}

TABLE II

Monte Carlo Yejo Predictions

compared with the yield predicted by the Poisson model in Fig. 8(a)-dd), and in tabular form in Table 11. The error of the models is calculated as the absolute difference between the predicted and simulated yield. The average and maximum errors for each of the four cases is given in Table IIl. In is seen that the average error for all four redundancies is less than $5 \%$, with maximum errors in the range of $8-11 \%$.

It is apparent from the Monte Carlo simulation data shown in Table 11 that the maximum yield is obtained for a redundancy factor of $r=1.5$. A simple calculation shows that the yield as predicted by (10) has a maximum at $r=0$. Hence. we would expect that the best predicted yield would occur at 
TABLE III

average and Maximum Errors of the Poisson Yield Models Versus Monte Carlo Simulation Results

\begin{tabular}{|c|c|c|c|c|}
\hline 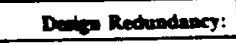 & $r=1.3$ & $r=1.5$ & $r=2.0$ & $r=2.5$ \\
\hline Averoge Emor (5) & 4.2 & 3.0 & 3.2 & 3.9 \\
\hline Mard Error (\$) & 8.6 & 10.8 & 10.9 & 10.8 \\
\hline
\end{tabular}

the minimum feasible redundancy, in this case $r=1.3$. We attribute this discrepancy to the fact that the Poisson yield model does not account for the effects of parasitic elements. These can cause a significant distortion in the frequency response, and are responsible for requirement of a greater than unity redundancy factor to meet the nominal response. The effect of missing taps then worsens this situation to the point that any missing tap of appreciable weight leads to a specification failure. We did investigate the use of a zero redundancy model for this case. This provided a more pessimistic yield prediction, but the errors when compared with the Monte Carlo simulations were higher.

\section{SUMmary AND CONCLUSIONS}

In this paper, we have predicted ACT-PTF yield from Poisson models. An equivalent circuit model was developed and four filters were designed to meet a typical specification with some degree of margin over that obtained with the minimum number of taps. Poisson yield models were developed assuming one redundant tap, and weighted by a uniform defect density distribution. The yield predicted by these models was calculated over the range of average defect densities over which the model has been reported to be accurate. A Monte Carlo yield simulation was then performed on the equivalent circuil for each of the defect densities. It was found that the one-redundancy Poisson model agreed with the Monte Carol simulation with an average error of better than $5 \%$, and maximum errors of about $10 \%$. In the prediction of the optimum redundancy, the Poisson model required the minimum redundancy, while the Monte Carlo simulations showed that the best yield over the range of defect densities considered occurs at a redundancy factor of 1.5. We attributed this discrepancy to the fact that the Poisson model neglects small parasitic effects, which can cause a specification deviation. The Monte Carlo model takes these into account, and thus requires some compensation in the form of redundant taps.

\section{ACKNOWLEDGMENT}

The authors gratefully acknowledge the help of Achankeng Leke in the derivation of the element values for the tap weighting circuits.

\section{REFERENCES}

11] R. L. Miller. C. E. Nothnick, and D. S. Bailey, Acoustic Charge Transpon: Device Technology and Applications. Norwood, MA: Artech House, 1992.

[2] C. H. Séquin and M. F. Tompsett, Charge Transfer Levices. New York: Academic Press Inc., 1975.
[3] S. D. Gaalema, R. J. Schwart, and R. L. Gunshor, "Acoustic surface wave interaction charge-coupled device," Appl. Phys. Lett., vol. 29, no. 2. pp. 82-82. July 1976 .

(4) M. J. Hoskins, H. Morkoc, and B. J. Hunsinger, "Charge ranspor by surface acoustic waves in GaAs," Appl. Phys. Letr., vol. 41, no. 4, pp. 332-34, Aug. 1982.

[5] W. J. Tanski, S. W. Merrit, R. N. Secks, and D. E. Cullen, "Heterojunction acoustic charge transport devices on GaAs," Appl. Phys. Lett., vol. 52, no. 1, pp. 18-19, Jan. 1988.

[6] F. Goodenough, "IC signal processor runs 45 billion MACs/s," Electronic Design, pp. 51-56. July 1992.

(7) J. S. Kenney, W. D. Hunt, and G. S. May. "Yield prediction of coustic charge transport transversal filters," in Proc. Int. Symp. Electron. Manufact. Technol... 1993, pp. 390-95.

[8] J. S. Kenney and W. D. Hunt, "A small-signal equivalent circuit model for helerostructure acoustic charge transpon devices," in Proc. Ultrasonics Symp., 1992, pp. 215-20.

[9] A. V. Oppenheim and R. W. Schafer, Discrete-Time Signal Processing. Englewood Cliffs, NJ: Prentice Hall, 1989, pp. 403-88.

[10] R. N. Bracewell, The Fourier Transform and lis Applications, 2nd ed., New York: McGraw-Hill, 1986.

[11] Libra ${ }^{\mathrm{TM}}$ is available from EEsof, Inc., 6501 Lindero Canyon Rd., Westlake Village, CA, 91362.

[12] C. H. Stapper, "Fact and fiction in yield modeling," Microelectronics J., vol. 20 , no. 1-2, pp. 129-51, 1989.

[13] J. A. Cunningham. The use and evaluation of yield models in integrated circuit manufacturing," IEEE Trans. Semicond. Manufact., vol. 3, no. 2. pp. 60-71, May 1990

[14] W. H. Hines and D. C. Mongomery, Probability and Sratistics in Engineering and Management Science. New York: Wiley, 1980.

[15] B. T. Murphy, "Cost-size optimization of monolithic integrated circuits," Proc. IEEE, vol. 52, pp. 1537-1545, Dec. 1964

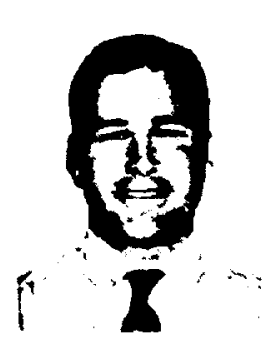

J. Stevenaon Kenney (S'84-M'85) was born in St. Louis, MO, in 1962. He received the B.S. and M.S. degrees from the Georgia Institute of Technology. Atlenta, GA, both in electrical engineering, in 1985 and 1990, respectively, where be is currenuly pursuing the Ph.D. degree.

Prior to retuming to graduate school full-time in 1992. be was employed by Electromagnetic Sciences, Inc. Scientific Atlanta, and SPC Electronics America, Inc., all of Norcross, Georgia. His technical interests include microwave devices, RF and microwave electronics, communication systems, SAW devices, and biomedical ulussound imaging systems.

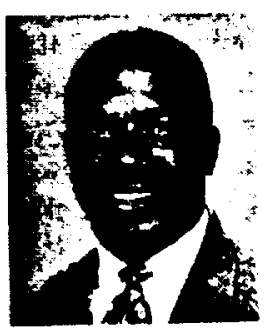

Gary S. May (S'85-M'90) received the B.S. degree in electrical engineering from the Georgis Institute of Technology in 1985, and the M.S. and Ph.D. degrees in electrical engineering and computer science from the University of Califomia at Berkeley, in 1987 and 1991, respectively.

He is currently an Assistant Professor in the School of Electrical and Computer Engineering and Microelectronics Research Center at the Georgia Institute of Technology. His research is in the field of computer-aided manufacturing of integrated circuits, and his interests include semiconductor process and equipment modeling. process simulation and control, automated process and equipment diagnosis, and yield modeling. He is currently an Associated Editor of IFEE Trunsactions on SEmiconductor Manufacturing. He was a National Science Foundation and an AT\&T Bell Laboralories graduate fellow, and has worked as a member of the technical staff at AT\&T Bell Leboratories in Murray Hill, NJ. He was National Chairperson of the National Sociery of Black Engineers (NSBE) from 1987 to 1989.

Dr. May is a National Science Foundation National Young Investigator. 


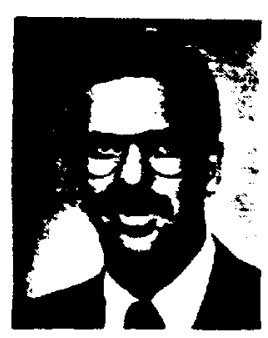

Wiltiam D. Hunt ( $M \cdot 83$ ) was born in Jackson. MS, on December 21, 1954. He received the B.S. degree from the University of Alabama. in 1976, the S.M degree from M.1.T., in 1980, and the Ph.D. degree from the University of Illinois, Champaign-Urbana, in 1987, all in electrical engineering.

He worked for Harris Corporation from 1976 to 1978, and for Bolt Beranek and Newman from 1980 to 1984, and joined the faculty of Georgia Tech in 1987. where he is currently an Associate Professor. His research interests include SAW and ACT devices, as well as transducers for biomedical ultrasound.

Dr. Hunt was a Rhodes Scholar Finalist in 1975, received a DuPont Young Faculty Award in 1988, and was named an National Science Foundation Young Investigator in 1989. In addition, he has been selected for Who's Who in the South and Soushwest and Who's Who in American Education. 